\title{
Phosphorylation at endothelial cell-cell junctions: Implications for VE-cadherin function
}

REVIEW

This article was published in the following Dove Press journal:

Cell Health and Cytoskeleton

15 May 2010

Number of times this article has been viewed

\section{Ilse Timmerman \\ Peter L Hordijk \\ Jaap D van Buul}

Department of Molecular Cell Biology, Sanquin Research and Landsteiner Laboratory, Academic Medical Center, University of Amsterdam, The Netherlands
Correspondence: Jaap D van Buul Sanquin Research and Landsteiner Laboratory, Academic Medical Center, University of Amsterdam, Plesmanlaan 125, 1066 CX Amsterdam,

The Netherlands

Tel $+3|-20-5| 23250$

Fax +3I-20-5 I 23474

Email j.vanbuul@sanquin.nl

\begin{abstract}
Endothelial cell-cell junctions are strictly regulated in order to control the barrier function of endothelium. Vascular endothelial (VE)-cadherin is one of the proteins that is crucial in this process. It has been reported that phosphorylation events control the function of VE-cadherin. This review summarizes the role of VE-cadherin phosphorylation in the regulation of endothelial cell-cell junctions and highlights how this affects vascular permeability and leukocyte extravasation.
\end{abstract}

Keywords: endothelium, VE-cadherin, junctions, barrier, phosphorylation

\section{Introduction}

The vascular endothelium is the inner lining of blood vessels and forms a physical barrier between the vessel lumen and surrounding tissue; controlling the extravasation of fluids, plasma proteins and leukocytes. Changes in the permeability of the endothelium are tightly regulated. Under basal physiological conditions, there is a continuous transfer of substances across the capillary beds. ${ }^{1}$ In addition the endothelium can mediate inducible, transient hyperpermeability in response to stimulation with inflammatory mediators, which takes place primarily in postcapillary venules. However, when severe, inflammation may result in dysfunction of the endothelial barrier in various parts of the vascular tree, including large veins, arterioles and capillaries. ${ }^{1,2}$ Dysregulated permeability is observed in various pathological conditions, such as tumor-induced angiogenesis, cerebrovascular accident and atherosclerosis. Two fundamentally different pathways regulate endothelial permeability, the transcellular and paracellular pathways. Solutes and cells can pass through the body of endothelial cells via the transcellular pathway, which includes vesicular transport systems, fenestrae, and biochemical transporters. ${ }^{3}$ The paracellular route is controlled by the coordinated opening and closing of endothelial junctions and thereby regulates traffic across the intercellular spaces between endothelial cells. ${ }^{4,5}$

Endothelial cells are connected by tight, gap and adherens junctions, of which the latter, and particularly the adherens junction component, vascular endothelial (VE)-cadherin, are of central importance for the initiation and stabilization of cell-cell contacts. ${ }^{6-8}$ Although multiple adhesion molecules are localized at endothelial junctions, blocking the adhesive function of VE-cadherin using antibodies is sufficient to disrupt endothelial junctions and to increase endothelial monolayer permeability both in vitro and in vivo. ${ }^{9-11}$ Like other cadherins, VE-cadherin mediates adhesion via homophilic, calcium-dependent interactions. ${ }^{6}$ 
This cell-cell adhesion is strengthened by binding of cytoplasmic proteins, the catenins, to the C-terminus of VE-cadherin. ${ }^{12}$ VE-cadherin can directly bind $\beta$-catenin and plakoglobin, which both associate with the actin binding protein $\alpha$-catenin. ${ }^{13}$ Initially, $\alpha$-catenin was thought to directly anchor cadherins to the actin cytoskeleton, but recently it became clear that $\alpha$-catenin cannot bind to both $\beta$-catenin and actin simultaneously. ${ }^{14,15}$ Data using purified proteins show that monomeric $\alpha$-catenin binds strongly to cadherin-bound $\beta$-catenin; in contrast to the dimer which has a higher affinity for actin filaments, indicating that $\alpha$-catenin might function as a molecular switch regulating cadherin-mediated cell-cell adhesion and actin assembly. ${ }^{14,15}$ Thus, interactions between the cadherin complex and the actin cytoskeleton are more complex than previously thought. Recently, Takeichi and colleagues reported that the actin binding protein EPLIN (epithelial protein lost in neoplasm) can associate with $\alpha$-catenin and thereby link the E-cadherin-catenin complex to the actin cytoskeleton. ${ }^{16}$ Although this study was performed in epithelial cells, an EPLIN-like molecule might serve as a bridge between the cadherin-catenin complex and the actin cytoskeleton in endothelial cells.

Next to $\beta$-catenin and plakoglobin, p120-catenin also binds directly to the intracellular tail of VE-cadherin. Numerous lines of evidence indicate that p120-catenin promotes VE-cadherin surface expression and stability at the plasma membrane. ${ }^{17,18}$ Different models are proposed that describe how p120-catenin regulates cadherin membrane dynamics, including the hypothesis that 120 -catenin functions as a 'cap' that prevents the interaction of VE-cadherin with the endocytic membrane trafficking machinery. ${ }^{19}$ In addition, p120-catenin might regulate VE-cadherin internalization through interactions with small GTPases. ${ }^{19}$ Cytoplasmic p120catenin, which is not bound to VE-cadherin, has been shown to decrease RhoA activity, elevate active Rac1 and Cdc42, and thereby is thought to regulate actin cytoskeleton organization and membrane trafficking. ${ }^{20,21}$

The intact cadherin-catenin complex is required for proper functioning of the adherens junction. Mutant forms of VE-cadherin which lack either the $\beta$-catenin, plakoglobin or p120 binding regions reduce the strength of cell-cell adhesion. ${ }^{13,22}$ Moreover, our own results showed that interfering with the interaction between $\alpha$-catenin and $\beta$-catenin, using a cell-permeable peptide which encodes the binding site in $\alpha$-catenin for $\beta$-catenin, resulted in an increased permeability of the endothelial monolayer. ${ }^{23}$ Several mechanisms may be involved in the regulation of the organization and function of the cadherin-catenin complex, including endocytosis of the complex, VE-cadherin cleavage and actin cytoskeleton reorganization. The remainder of this review primarily focuses on the role of tyrosine phosphorylation in the control of VE-cadherin-mediated cell-cell adhesion.

\section{Regulation of the adhesive function of VE-cadherin by tyrosine phosphorylation}

It is a widely accepted concept that tyrosine phosphorylation of components of the VE-cadherin-catenin complex correlates with the weakening of cell-cell adhesion.,24 One of the first reports that supported this idea showed that the level of phosphorylation of VE-cadherin was high in loosely confluent endothelial cells, but low in tightly confluent monolayers, when intercellular junctions are stabilized. ${ }^{25}$ In addition, several conditions that induce tyrosine phosphorylation of adherens junction components, like $\mathrm{v}$-Src transformation and inhibition of phosphatase activity by pervanadate, have been shown to shift cell-cell adhesion from a strong to a weak state. ${ }^{26-28}$ More physiologically relevant; permeability-increasing agents such as histamine, tumor necrosis factor- $\alpha$ (TNF- $\alpha)$, thrombin, platelet-activating factor (PAF) and vascular endothelial growth factor (VEGF) increase tyrosine phosphorylation of various components of the cadherin-catenin complex. ${ }^{29-34}$

Although most of the data showing a correlation between tyrosine phosphorylation and the weakening of cell-cell adhesion are obtained using cultured cells, some studies have shown that VE-cadherin can also be phosphorylated in vivo. Systemic VEGF administration into healthy, but not in Src kinase-deficient mice, was shown to induce an increased permeability of the vasculature in the heart. ${ }^{35}$ According to the authors, this increased vascular permeability was associated with higher tyrosine phosphorylation levels of VE-cadherin and $\beta$-catenin and a disruption of the VE-cadherin- $\beta$-catenin association. ${ }^{35}$ Others detected phosphorylated VE-cadherin in mouse lung, uterus and to a lesser extent in ovary extracts. ${ }^{36}$ While VE-cadherin was only weakly phosphorylated in resting vasculature in the female reproductive organs, higher phosphorylation states were detected during hormonally induced angiogenesis. ${ }^{36}$

A general idea has emerged that tyrosine phosphorylation of the VE-cadherin complex leads to the uncoupling of VE-cadherin from the actin cytoskeleton through dissociation of catenins from the cadherin. However, 
partly contradictory results were obtained in vitro for the effect of tyrosine phosphorylation on the molecular organization of the VE-cadherin-catenin complex. Cell density-dependent and pervanadate-induced increases in tyrosine phosphorylation were both found to go along with increased binding of p120- and $\beta$-catenin to VE-cadherin. These two catenins were largely replaced by plakoglobin when the phosphotyrosine content of VE-cadherin was reduced. ${ }^{25}$ Increased tyrosine phosphorylation correlated with a reduced binding of p120- and $\beta$-catenin to VE-cadherin in response to both thrombin and VEGF stimulation in studies of Rabiet et $\mathrm{al}^{34}$ and Monaghan-Benson et $\mathrm{al}^{37}$ whereas others have reported that the composition of the cadherin-catenin complex remained unaltered during stimulation with various permeabilityinducing agents. ${ }^{29-31}$ Potter and colleagues studied the effect of phosphorylation of specific VE-cadherin tyrosine residues on cadherin-catenin complex organization. ${ }^{38}$ They showed that replacement of the tyrosines 658 and 731 of VE-cadherin by glutamate, which is used to mimic the charge effect of phosphorylation, results in a loss of binding of p120 and $\beta$-catenin, respectively. Expression of these mutants of VE-cadherin in Chinese hamster ovary (CHO) cells led to an inhibition of cell barrier function. Interestingly, the authors also showed, (by using immobilized VE-cadherin-Fc fusion proteins) that these mutants did not affect the adhesive function of VE-cadherin. However, based on this study, tyrosine phosphorylation of residues Y658 and Y731 is thought to negatively regulate VE-cadherin-mediated cell-cell adhesion. ${ }^{38}$ In line with this, phosphorylation of VE-cadherin at both Y658 and Y731 was recently found to be increased in response to VEGF stimulation in human pulmonary microvessel endothelial cells (HMVECs), which was critical for the VEGF-induced vascular permeability. ${ }^{37} \mathrm{~A}$ schematic overview of the signals leading to VE-cadherin phosphorylation is shown in Figure $1 \mathrm{~A}$.

The functional significance of the Y658 and Y731 residues of VE-cadherin is further underscored by Allingham and colleagues, ${ }^{39}$ who reported that the adhesion of leukocytes to endothelial cells via intercellular adhesion molecule-1 (ICAM-1) induced VE-cadherin tyrosine phosphorylation at these specific tyrosine residues. In addition, human umbilical vein endothelial cells (HUVECs) overexpressing a mutated form of VE-cadherin, (in which a single tyrosine was substituted for phenylalanine at either residue 658 or 731) showed reduced migration of leukocytes across the endothelial monolayer. ${ }^{39}$ These findings were extended by a study which showed that overexpression of p120-catenin resulted in decreased transmigration of leukocytes. The authors suggested that this was caused by a p120-catenin-induced repression of VE-cadherin phosphorylation at $\mathrm{Y} 658,{ }^{40}$-the residue corresponding to the p120-catenin binding site. ${ }^{38}$ In a similar study, expression of nonphosphorylatable mutants of VE-cadherin for either tyrosine 645,731 or 733 in VE-cadherin-null endothelial cells resulted in less efficient transendothelial migration (TEM) of lymphocytes compared to conditions when wild-type VE cadherin was re-expressed. ${ }^{41}$ In contrast to the reports of Allingham et $\mathrm{al}^{39}$ and Alcaide et $\mathrm{al}^{40}{ }^{40}$ these authors demonstrated that the expression of the VE-cadherin construct with a single point mutation in Y658 did not have any effect on lymphocyte TEM. These discrepancies concerning the importance of different tyrosine residues for TEM might, in part, reflect differences in experimental conditions, such as different types of endothelial cells (HUVEC versus microvascular endothelial cells (MVEC) and VE-cadherin-null EC) and leukocytes (neutrophils versus lymphocytes). However, the results demonstrate that tyrosine phosphorylation of VEcadherin is required for efficient transmigration of leukocytes. This suggests that VE-cadherin-mediated cell-cell contacts are not just pushed open by the migrating leukocytes, but play a more active role in the transmigration process. A schematic overview of leukocyte adhesion-induced signals leading to VE-cadherin phosphorylation is shown in Figure 1B.

\section{Tyrosine phosphorylation of VE-cadherin-associated catenins}

Changes in VE-cadherin-mediated cell-cell adhesion are often not only correlated with alterations in tyrosine phosphorylation of the cadherin itself, but of VE-cadherin-associated catenins as well. $\beta$-catenin, p120-catenin and plakoglobin can be tyrosine phosphorylated by the same permeability-increasing agents that induce VE-cadherin phosphorylation. ${ }^{29-31}$ Therefore, catenin phosphorylation is also generally associated with the disruption of cell-cell junctions; however, the direct relationship between phosphorylation of the catenins and reduced VE-cadherin-mediated cell-cell adhesion has remained elusive. Increased tyrosine phosphorylation of catenins has been correlated with a reduction in their affinity for the cadherin cytoplasmic tail, ${ }^{34,37}$ while other data indicate that phosphorylation of catenins on the tyrosines did not result in disassembly of the cadherin-catenin complex. ${ }^{25,29-31}$ However, in these studies phosphorylation of the catenins was induced in parallel with the phosphorylation of VE-cadherin. Therefore, it is difficult to conclude that; even if there was a 


\section{A Permeability-inducing agents}

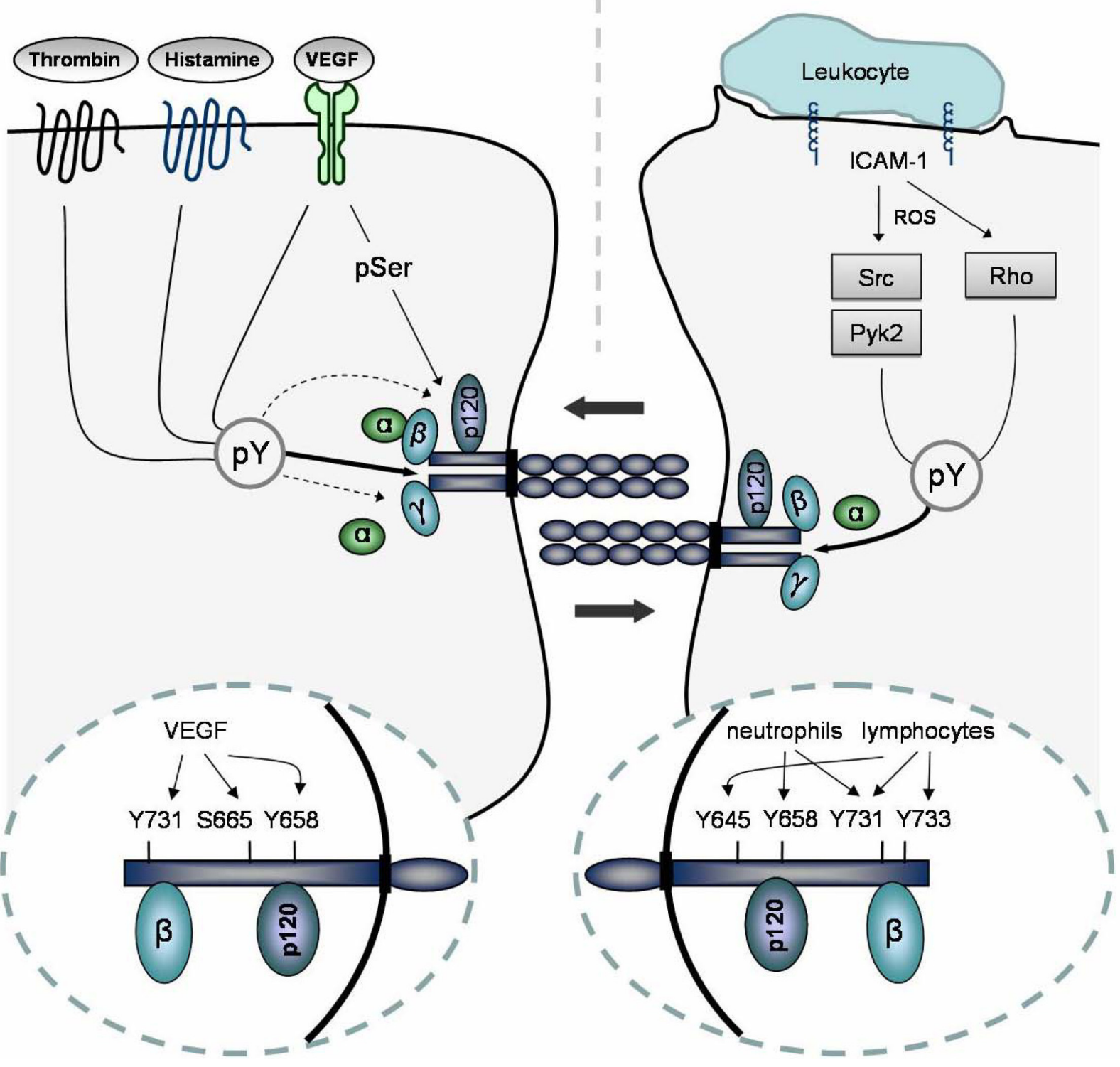

Figure I Regulation of the integrity of endothelial cell-cell contacts by phosphorylation of VE-cadherin.

Notes: A) Permeability-inducing agents such as thrombin, histamine and VEGF, induce tyrosine phosphorylation (PY) of VE-cadherin and the associated catenins. Although the specific consequences of catenin tyrosine phosphorylation in endothelial cells are still unknown,VE-cadherin tyrosine phosphorylation results in opening of the cell-cell junctions (indicated by arrows) and enhanced vascular permeability. How tyrosine phosphorylation affects VE-cadherin adhesiveness is not yet well understood; disrupted binding of catenins, which link the cadherin to the actin cytoskeleton, may be involved.VEGF induces phosphorylation of VE-cadherin at specific residues, $Y 658$ and $Y 73$, which have been reported to regulate $\mathrm{pI} 20$-catenin and $\beta$-catenin binding, respectively. Moreover,VEGF stimulation results in serine phosphorylation (pSer) ofVE-cadherin, specifically at residue S665, which leads to its endocytosis. B) Adhesion of leukocytes to endothelial cells via ICAM-I increases endothelial permeability by inducing phosphorylation of VE-cadherin on tyrosine residues. Essential mediators, such as the kinases Pyk2 and Src, and signaling routes involving reactive oxygen species (ROS) and Rho, have been shown to act downstream of ICAM-I. Different tyrosine residues within the cytoplasmic domain of VE-cadherin are involved in the extravasation of neutrophils and lymphocytes, including Y658 and Y73।. ( $\beta$ : $\beta$-catenin, $\alpha$ : $\alpha$-catenin, $\gamma$ : $\gamma$-catenin/plakoglobin).

dissociation of catenins from VE-cadherin, phosphorylation of catenins is the initiating event.

The consequences of catenin phosphorylation on E-cadherin function have been studied in more detail. Overall, most of the studies in epithelial cells suggest that tyrosine phosphorylation of $\beta$-catenin results in its dissociation from E-cadherin. ${ }^{42-45}$ Several specific tyrosine residues in $\beta$-catenin that are relevant for the regulation of cell-cell contacts are identified. The binding of $\beta$-catenin to E-cadherin was found to be disrupted by phosphorylation of $\beta$-catenin on Y489 or Y 654. ${ }^{43,44}$ In addition, phosphorylation of $\beta$-catenin on Y142 negatively regulates binding to $\alpha$-catenin. ${ }^{46,47}$ For p120-catenin, more contradictory studies have been published. ${ }^{48}$ Phosphorylation of the p120-Y217 residue has 
been reported to negatively regulate E-cadherin function, since v-Src kinase induced effects on cadherin function in L-cells were partially reversed by the mutation of this tyrosine residue to phenylalanine. ${ }^{49}$ In a similar study in A431 cells, expression of a non-phosphorylatable p120-catenin construct, in which seven other tyrosine residues were mutated, did not affect E-cadherin adhesiveness. ${ }^{50}$ Interestingly, several studies even demonstrate that tyrosine phosphorylation of p120-catenin correlates with an increased affinity for E-cadherin. . $^{27,44,47,51}$ These contrasting results may reflect differences in experimental conditions; eg, the data were obtained in different cell lines. Moreover, the discrepancies might also be the result of a complex regulation of p120-catenin phosphorylation, in which phosphorylation of specific tyrosine residues of p120-catenin may have varying effects on the association of p120-catenin with the cadherin.

In conclusion, the precise consequences of catenin phosphorylation for vascular permeability are still unknown; however, the data from studies using epithelial cells thus far suggest that tyrosine phosphorylation of catenins can influence the adhesiveness of E-cadherin. While regulation of the affinity between cadherins and catenins by tyrosine phosphorylation can of course differ, depending on cell type and cadherin specificities, these data suggest that tyrosine phosphorylation of catenins may also play an important role in regulation of VE-cadherin-mediated cell-cell adhesion.

\section{Regulation of VE-cadherin function by serine phosphorylation}

In addition to tyrosine phosphorylation, serine phosphory lation of VE-cadherin has been reported to be involved in the regulation of the endothelial barrier function. ${ }^{52}$ Phosphorylation of a specific serine residue within the intracellular domain of VE-cadherin, Ser665, leads to the endocytosis of VE-cadherin..$^{52}$ Phosphorylation of residue Ser665 was induced upon VEGF stimulation, through a signaling pathway that involves the Src-dependent activation of the guanine exchange factor (GEF) Vav2 and the consequent activation of Rac1. Subsequently, phosphorylation of VE-cadherin at the serine residue recruits $\beta$-arrestin 2 , thereby promoting clathrin-dependent internalization of VE-cadherin. Interestingly, the Ser665 site is adjacent to the binding region of p120-catenin, which has been shown to stabilize VE-cadherin expression at the plasma membrane by preventing its clathrin-mediated endocytosis. ${ }^{52,53}$ Therefore, Gavard and Gutkind suggest that the association of p120-catenin with VE-cadherin may regulate VE-cadherin phosphorylation at the serine residue and its interaction with $\beta$-arrestin $2 .^{52}$ Thus, serine phosphorylation and tyrosine phosphorylation may act in concert to coordinate the dynamic disassembly and reassembly of adherens junctions (Figure 1A).

\section{VE-cadherin regulation by protein tyrosine phosphatases (PTP)}

Phosphorylation of adherens junctions is regulated by a dynamic balance between the activity of kinases and phosphatases.$^{54}$ Data from our studies, and others, show that Src-kinase and proline-rich tyrosine kinase (Pyk) 2 are both able to increase tyrosine phosphorylation levels of junctional proteins, including VE-cadherin and $\beta$-catenin, resulting in weakening of the endothelial junctions. ${ }^{35,39,55}$ In addition, the reduced activity of tyrosine phosphatases may promote tyrosine phosphorylation. ${ }^{56}$ Several phosphatases have been described to play an important role in the regulation of VE-cadherin function.

Density-enhanced phosphatase-1 (DEP-1)/CD148 regulates VE-cadherin-mediated cell-cell contacts through the VEGF receptor 2 (VEGFR-2). ${ }^{57}$ In epithelial cells, DEP-1 is involved in the maintenance of the barrier function through the regulation of tight junctions. ${ }^{58}$ The vascular endothelial protein tyrosine phosphatase (VE-PTP) interacts with the extracellular domain of VE-cadherin and supports VE-cadherin function in protecting endothelial integrity. ${ }^{59,60}$ Interestingly, leukocyte adhesion or VEGF treatment induces a rapid dissociation of VE-PTP from VE-cadherin, followed by an increased leukocyte migration across the endothelial monolayer and increased permeability. The dissociation of VE-PTP from VE-cadherin results in increased levels of tyrosine phosphorylation of not only VE-cadherin, but also of $\beta$-catenin and plakoglobin. Again, these studies show that tyrosine phosphorylation of VE-cadherin results in an inhibition of endothelial barrier function.

The SH2 domain-containing protein tyrosine phospha tase 2 (SHP2) has been implicated in the regulation of endothelial cell-cell junctions upon thrombin stimulation. ${ }^{61}$ According to the authors, thrombin stimulation results in a dissociation of SHP2 from $\beta$-catenin. This would result in increased phosphotyrosine levels of $\beta$ catenin, followed by a dissociation of $\beta$-catenin from VE-cadherin and a loss of barrier function. Thus, just as several tyrosine kinases are involved in the regulation of VE-cadherin phosphorylation, different phosphatases are important as well. This suggests that various signaling 
pathways exist that control VE-cadherin phosphorylation and endothelial barrier integrity.

\section{ROS-mediated VE-cadherin phosphorylation}

Although different protein phosphatases and kinases regulate VE-cadherin function by affecting its phosphorylation, it is less clear how these proteins are activated or triggered.

Several studies have shown that reactive oxygen species (ROS) can effectively inhibit phosphatase activity. ${ }^{62}$ By oxidation of crucial cysteine residues, the phosphatases loose their active conformation and return to their inactive state. ${ }^{56,63,64}$ ROS are also generated in endothelial cells and can serve as potential signaling mediators. ${ }^{65}$ Introducing active mutants of Rac1 increases the production of ROS in endothelial cells. ${ }^{66}$ This results in a loss of VE-cadherin-mediated cell-cell junctions and an increase in the permeability of the endothelial monolayer. Moreover, disrupting VE-cadherin-mediated cell-cell junctions by treating the endothelial monolayer with an anti-VE-cadherin antibody also induced the production of ROS..$^{55}$ Detailed analysis of this action by high resolution confocal microscopy showed that ROS production precedes the loss of cell-cell contacts. The induction of ROS by antibody-induced loss of VEcadherin-mediated cell-cell contacts increased the tyrosine phosphorylation levels of Pyk2. The activation of this kinase has been shown to be redox-sensitive. ${ }^{67}$ In addition, $\beta$-catenin tyrosine phosphorylation was increased upon the loss of VEcadherin-mediated cell-cell junctions, which was prevented by the overexpression of a dominant negative mutant of Pyk2. ${ }^{55}$ In line with this, the dominant, negative mutant of Pyk2 prevented the antibody-induced loss of VE-cadherinmediated cell-cell contacts. However, these data do not exclude that ROS can directly act on a phosphatase by inhibiting its activity and, as a result, shift the balance to increased phosphorylation. Interestingly, scavenging ROS resulted in increased barrier function in non-stimulated endothelial monolayers. ${ }^{55}$ In addition, overall tyrosine phosphorylation levels were reduced when ROS was scavenged (JDvB, unpublished results). Thus, Rac1-induced ROS production may indirectly increase kinase activity by inhibiting phosphatases. This hypothesis is recently underscored by a publication by Monaghan-Benson and Burridge. They showed that VEGF leads to a Rac1-mediated generation of ROS, which, in turn, elevates the tyrosine phosphorylation of VE-cadherin and $\beta$ catenin, negatively regulating adherens junction integrity. ${ }^{37} \mathrm{It}$ is interesting to note that Rac1-wt and VE-cadherin co-localize at cell-cell junctions in endothelial cells and therefore are in close proximity to each other (Figure 2). Whether ROS is generated at or near cell-cell junctions is unknown and requires further investigation.

The generation of ROS is controlled by the NADPHoxidase (NOX) protein complex in many different cell types. ${ }^{68}$ ROS levels in endothelial cells are more than 100 -fold less than the levels of neutrophils. ${ }^{69}$ Nevertheless, all major components of the NADPH-complex, including the NADPH-oxidase complex catalytic units NOX2 and NOX4, are expressed in endothelial cells. ${ }^{70}$ Interestingly, Chen and co-workers showed that NOX4 is critical in the regulation of protein tyrosine phosphatase 1B in endothelial cells. ${ }^{71}$ Nevertheless, for many pathways that involve the generation of ROS, it is not clear which of these NOX-proteins are responsible for the ROS production in endothelial cells. Future research should clarify this point.

\section{Concluding remarks}

VE-cadherin is an endothelial-specific adhesion molecule, which is crucial for the maintenance and control of endothelial cell-cell contacts. Much progress has been
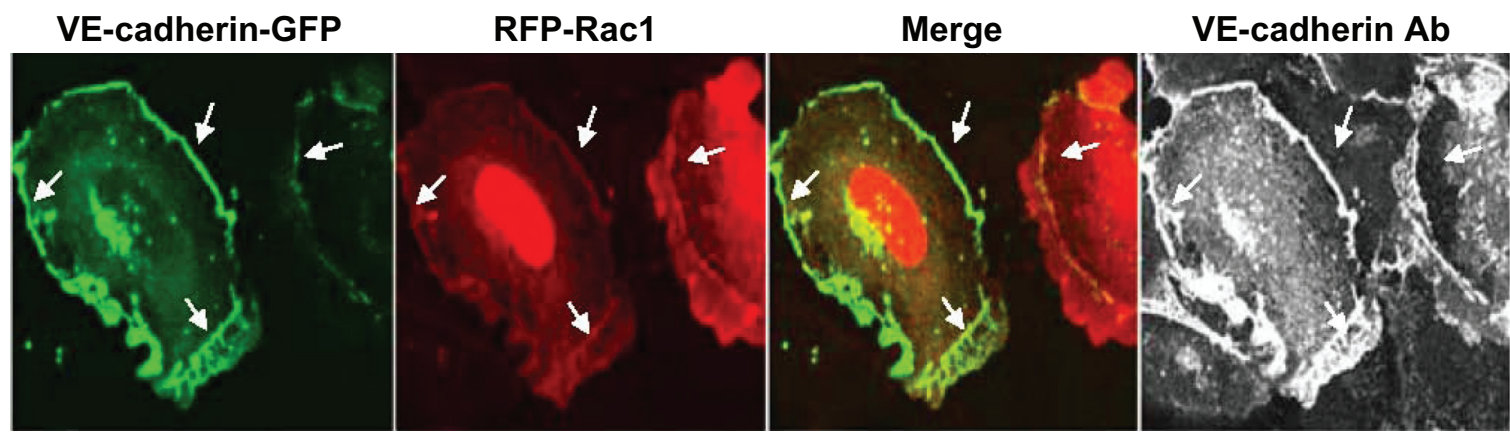

Figure 2 Racl co-localizes with VE-cadherin at endothelial cell-cell junctions.

Notes: HUVECs were transiently transfected with VE-cadherin-GFP (green) and RFP-Racl-wildtype (red). Merge shows the co-localization of the green and red signal. Most right image shows endogenous VE-cadherin at cell-cell junctions (white). 
made in our understanding of the molecular mechanisms regulating VE-cadherin function. Recent studies have identified specific tyrosine residues within the cytoplasmic tail of VE-cadherin that regulate vascular permeability and extravasation of leukocytes. Moreover, several kinases and phosphatases were found to be involved in the regulation of the adhesive strength of VE-cadherin. However, further work is required to address important questions such as; how tyrosine phosphorylation of the VE-cadherin-catenin complex leads to changes in the integrity of cell-cell junctions. Furthermore, the relevance of tyrosine phosphorylation of VE-cadherin in vivo remains elusive. While VE-cadherin can be phosphorylated in angiogenic and ischemic conditions in mouse tissues, ${ }^{35,36}$ it is still not clear whether VE-cadherin phosphorylation is involved in the basal, dynamic regulation of the endothelial barrier in vivo or whether it only plays a role in pathological conditions.

Interestingly, VE-cadherin has been recently shown to indirectly control tight junction organization. ${ }^{72} \mathrm{VE}$-cadherin expression and clustering was demonstrated to be required for transcriptional upregulation of the tight junction adhesive protein claudin-5. This direct positive relationship between adherens junctions and tight junctions may have important functional consequences and may further explain why interfering with only VE-cadherin function has marked effects on junctional organization and vascular permeability in general.

Ultimately, elucidating the molecular mechanisms by which VE-cadherin function is regulated in endothelial cell-cell junctions will help to identify new therapeutic targets for the treatment of many diseases that are accompanied by increased vascular permeability.

\section{Acknowledgment}

Jaap D van Buul is supported by the Dutch Heart Foundation (grant no. 2005T039) and NWO Veni grant 916.76.053. Ilse Timmerman is supported by Sanquin Research.

\section{Disclosures}

The authors report no conflicts of interest relevant to this research.

\section{References}

1. Pappenheimer JR. Passage of molecules through capillary wals. Physiol Rev. 1953;33(3):387-423.

2. Aird WC. Phenotypic heterogeneity of the endothelium: I. Structure, function, and mechanisms. Circ Res. 2007;100(2):158-173.

3. Dvorak AM, Kohn S, Morgan ES, Fox P, Nagy JA, Dvorak HF. The vesiculo-vacuolar organelle (VVO): a distinct endothelial cell structure that provides a transcellular pathway for macromolecular extravasation. J Leukoc Biol. 1996;59(1):100-115.
4. Dejana E, Orsenigo F, Lampugnani MG. The role of adherens junctions and VE-cadherin in the control of vascular permeability. $J$ Cell Sci. 2008;121(Pt 13):2115-2122.

5. Muller WA. Migration of leukocytes across endothelial junctions: some concepts and controversies. Microcirculation. 2001;8(3):181-193.

6. Breviario F, Caveda L, Corada M, et al. Functional properties of human vascular endothelial cadherin (7B4/cadherin-5), an endothelium-specific cadherin. Arterioscler Thromb Vasc Biol. 1995;15(8):1229-1239.

7. Crosby CV, Fleming PA, Argraves WS, et al. VEcadherin is not required for the formation of nascent blood vessels but acts to prevent their disassembly. Blood. 2005;105(7):2771-2776.

8. Matsuyoshi N, Toda K, Horiguchi Y, Tanaka T, Nakagawa S, Takeichi M, et al. In vivo evidence of the critical role of cadherin-5 in murine vascular integrity. Proc Assoc Am Physicians. 1997;109(4):362-371.

9. Corada M, Mariotti M, Thurston G, et al. Vascular endothelial-cadherin is an important determinant of microvascular integrity in vivo. Proc Natl Acad Sci U S A. 1999;96(17):9815-9820.

10. Gotsch U, Borges E, Bosse R, Boggemeyer E, Simon M, Mossmann H, et al. VE-cadherin antibody accelerates neutrophil recruitment in vivo. J Cell Sci. 1997;110( Pt 5):583-588.

11. van Buul JD, Voermans C, van dB V, et al. Migration of human hematopoietic progenitor cells across bone marrow endothelium is regulated by vascular endothelial cadherin. J Immunol. 2002;168(2):588-596.

12. Kemler R. From cadherins to catenins: cytoplasmic protein interactions and regulation of cell adhesion. Trends Genet. 1993;9(9):317-321.

13. Navarro P, Caveda L, Breviario F, Mandoteanu I, Lampugnani MG, Dejana E. Catenin-dependent and -independent functions of vascular endothelial cadherin. J Biol Chem. 1995;270(52):30965-30972.

14. Drees F, Pokutta S, Yamada S, Nelson WJ, Weis WI. Alpha-catenin is a molecular switch that binds E-cadherin-beta-catenin and regulates actin-filament assembly. Cell. 2005;123(5):903-915.

15. Yamada S, Pokutta S, Drees F, Weis WI, Nelson WJ. Deconstructing the cadherin-catenin-actin complex. Cell. 2005;123(5):889-901.

16. Abe K, Takeichi M. EPLIN mediates linkage of the cadherin catenin complex to F-actin and stabilizes the circumferential actin belt. Proc Natl Acad Sci U S A. 2008;105(1):13-19.

17. Davis MA, Ireton RC, Reynolds AB. A core function for $\mathrm{p} 120$-catenin in cadherin turnover. $J$ Cell Biol. 2003;163(3):525-534.

18. Xiao K, Allison DF, Buckley KM, et al. Cellular levels of p120 catenin function as a set point for cadherin expression levels in microvascular endothelial cells. J Cell Biol. 2003;163(3):535-545.

19. Xiao K, Oas RG, Chiasson CM, Kowalczyk AP. Role of p120-catenin in cadherin trafficking. Biochim Biophys Acta. 2007;1773(1):8-16.

20. Anastasiadis PZ, Moon SY, Thoreson MA, et al. Inhibition of RhoA by p120 catenin. Nat Cell Biol. 2000;2(9):637-644.

21. Noren NK, Liu BP, Burridge K, Kreft B. p120 catenin regulates the actin cytoskeleton via Rho family GTPases. $J$ Cell Biol. 2000;150(3):567-580.

22. Ferber A, Yaen C, Sarmiento E, Martinez J. An octapeptide in the juxtamembrane domain of VE-cadherin is important for p120ctn binding and cell proliferation. Exp Cell Res. 2002;274(1):35-44.

23. van Buul JD, van Alphen FP, Hordijk PL. The presence of alpha-catenin in the VE-cadherin complex is required for efficient transendothelial migration of leukocytes. Int J Biol Sci. 2009;5(7):695-705.

24. Vestweber D. VE-cadherin: the major endothelial adhesion molecule controlling cellular junctions and blood vessel formation. Arterioscler Thromb Vasc Biol. 2008;28(2):223-232.

25. Lampugnani MG, Corada M, Andriopoulou P, Esser S, Risau W, Dejana E. Cell confluence regulates tyrosine phosphorylation of adherens junction components in endothelial cells. J Cell Sci. 1997;110 (Pt 17):2065-2077.

26. Hamaguchi M, Matsuyoshi N, Ohnishi Y, Gotoh B, Takeichi M, Nagai Y. p60v-src causes tyrosine phosphorylation and inactivation of the $\mathrm{N}$-cadherin-catenin cell adhesion system. EMBO J. 1993;12(1):307-314.

27. Kinch MS, Clark GJ, Der CJ, Burridge K. Tyrosine phosphorylation regulates the adhesions of ras-transformed breast epithelia. J Cell Biol. 1995;130(2):461-471. 
28. Matsuyoshi N, Hamaguchi M, Taniguchi S, Nagafuchi A, Tsukita S, Takeichi M. Cadherin-mediated cell-cell adhesion is perturbed by v-Src tyrosine phosphorylation in metastatic fibroblasts. J Cell Biol. 1992;118(3):703-714.

29. Andriopoulou P, Navarro P, Zanetti A, Lampugnani MG, Dejana E. Histamine induces tyrosine phosphorylation of endothelial cellto-cell adherens junctions. Arterioscler Thromb Vasc Biol. 1999;19(10):2286-2297.

30. Angelini DJ, Hyun SW, Grigoryev DN, et al. TNF-alpha increases tyrosine phosphorylation of vascular endothelial cadherin and opens the paracellular pathway through fyn activation in human lung endothelia. Am J Physiol Lung Cell Mol Physiol. 2006;291(6): L1232-L1245.

31. Esser S, Lampugnani MG, Corada M, Dejana E, Risau W. Vascular endothelial growth factor induces VE-cadherin tyrosine phosphorylation in endothelial cells. J Cell Sci. 1998;111(Pt 13):1853-1865.

32. Hudry-Clergeon H, Stengel D, Ninio E, Vilgrain I. Platelet-activating factor increases VE-cadherin tyrosine phosphorylation in mouse endothelial cells and its association with the PtdIns3'-kinase. FASEB J. 2005;19(6):512-520.

33. Nwariaku FE, Liu Z, Zhu X, Turnage RH, Sarosi GA, Terada LS. Tyrosine phosphorylation of vascular endothelial cadherin and the regulation of microvascular permeability. Surgery. 2002;132(2):180-185.

34. Rabiet MJ, Plantier JL, Rival Y, Genoux Y, Lampugnani MG, Dejana E. Thrombin-induced increase in endothelial permeability is associated with changes in cell-to-cell junction organization. Arterioscler Thromb Vasc Biol. 1996;16(3):488-496.

35. Weis S, Shintani S, Weber A, et al. Src blockade stabilizes a Flk/cadherin complex, reducing edema and tissue injury following myocardial infarction. J Clin Invest. 2004;113(6):885-894.

36. Lambeng N, Wallez Y, Rampon C, et al. Vascular endothelial-cadherin tyrosine phosphorylation in angiogenic and quiescent adult tissues. Circ Res. 2005;96(3):384-391.

37. Monaghan-Benson E, Burridge $\mathrm{K}$. The regulation of vascular endothelial growth factor-induced microvascular permeability requires Rac and reactive oxygen species. $J$ Biol Chem. 2009;284(38):25602-25611.

38. Potter MD, Barbero S, Cheresh DA. Tyrosine phosphorylation of VE-cadherin prevents binding of p120- and beta-catenin and maintains the cellular mesenchymal state. $J$ Biol Chem. 2005;280(36):31906-31912.

39. Allingham MJ, van Buul JD, Burridge K. ICAM-1-mediated, Src- and Pyk2-dependent vascular endothelial cadherin tyrosine phosphorylation is required for leukocyte transendothelial migration. $J$ Immunol. 2007;179(6):4053-4064.

40. Alcaide P, Newton G, Auerbach S, Sehrawat S, Mayadas TN, Golan DE, et al. p120-catenin regulates leukocyte transmigration through an effect on VE-cadherin phosphorylation. Blood. 2008;112(7): 2770-2779.

41. Turowski P, Martinelli R, Crawford R, et al. Phosphorylation of vascular endothelial cadherin controls lymphocyte emigration. J Cell Sci. 2008;121(Pt 1):29-37.

42. Huber AH, Weis WI. The structure of the beta-catenin/E-cadherin complex and the molecular basis of diverse ligand recognition by beta-catenin. Cell. 2001;105(3):391-402.

43. Lilien J, Balsamo J. The regulation of cadherin-mediated adhesion by tyrosine phosphorylation/dephosphorylation of beta-catenin. Curr Opin Cell Biol. 2005;17(5):459-465.

44. Roura S, Miravet S, Piedra J, Garcia dH, Dunach M. Regulation of E-cadherin/Catenin association by tyrosine phosphorylation. $J$ Biol Chem. 1999;274(51):36734-36740.

45. Winter MC, Shasby S, Shasby DM. Compromised E-cadherin adhesion and epithelial barrier function with activation of $\mathrm{G}$ protein-coupled receptors is rescued by Y-to-F mutations in beta-catenin. Am J Physiol Lung Cell Mol Physiol. 2008;294(3):L442-L448.

46. Aberle H, Schwartz H, Hoschuetzky H, Kemler R. Single amino acid substitutions in proteins of the armadillo gene family abolish their binding to alpha-catenin. J Biol Chem. 1996;271(3):1520-1526.
47. Piedra J, Miravet S, Castano J, et al. p120 Catenin-associated Fer and Fyn tyrosine kinases regulate beta-catenin Tyr-142 phosphorylation and beta-catenin-alpha-catenin Interaction. Mol Cell Biol. 2003;23(7):2287-2297.

48. Alema S, Salvatore AM. p120 catenin and phosphorylation: Mechanisms and traits of an unresolved issue. Biochim Biophys Acta. 2007;1773(1):47-58.

49. Ozawa M, Ohkubo T. Tyrosine phosphorylation of p120(ctn) in $\mathrm{V}$-Src transfected $\mathrm{L}$ cells depends on its association with E-cadherin and reduces adhesion activity. $J$ Cell Sci. 2001;114(Pt 3): $503-512$.

50. Mariner DJ, Davis MA, Reynolds AB. EGFR signaling to p120-catenin through phosphorylation at Y228. J Cell Sci. 2004;117(Pt 8):1339-1350.

51. Calautti E, Cabodi S, Stein PL, Hatzfeld M, Kedersha N, Paolo DG. Tyrosine phosphorylation and Src family kinases control keratinocyte cell-cell adhesion. J Cell Biol. 1998;141(6):1449-1465.

52. Gavard J, Gutkind JS. VEGF controls endothelial-cell permeability by promoting the beta-arrestin-dependent endocytosis of VE-cadherin. Nat Cell Biol. 2006;8(11):1223-1234.

53. Xiao K, Garner J, Buckley KM, et al. p120-catenin regulates clathrin-dependent endocytosis of VE-cadherin. Mol Biol Cell. 2005;16(11):5141-5151.

54. Brady-Kalnay SM, Tonks NK. Protein tyrosine phosphatases as adhesion receptors. Curr Opin Cell Biol. 1995;7(5):650-657.

55. van Buul JD, Anthony EC, Fernandez-Borja M, Burridge K, Hordijk PL. Proline-rich tyrosine kinase 2 (Pyk2) mediates vascular endothelialcadherin-based cell-cell adhesion by regulating beta-catenin tyrosine phosphorylation. J Biol Chem. 2005;280(22):21129-21136.

56. Chiarugi P. PTPs versus PTKs: the redox side of the coin. Free Radic Res. 2005;39(4):353-364.

57. Grazia LM, Zanetti A, Corada M, et al. Contact inhibition of VEGF-induced proliferation requires vascular endothelial cadherin, beta-catenin, and the phosphatase DEP-1/CD148. J Cell Biol. 2003;161(4):793-804.

58. Sallee JL, Burridge K. Density-enhanced phosphatase 1 regulates phosphorylation of tight junction proteins and enhances barrier function of epithelial cells. J Biol Chem. 2009;284(22):14997-15006.

59. Nawroth R, Poell G, Ranft A, et al. VE-PTP and VE-cadherin ectodomains interact to facilitate regulation of phosphorylation and cell contacts. EMBO J. 2002;21(18):4885-4895.

60. Nottebaum AF, Cagna G, Winderlich M, et al. VE-PTP maintains the endothelial barrier via plakoglobin and becomes dissociated from VE-cadherin by leukocytes and by VEGF. J Exp Med. 2008;205(12):2929-2945.

61. Ukropec JA, Hollinger MK, Salva SM, Woolkalis MJ. SHP2 association with VE-cadherin complexes in human endothelial cells is regulated by thrombin. J Biol Chem. 2000;275(8):5983-5986.

62. Groen A, Overvoorde J, van der WT, den Hertog J. Redox regulation of dimerization of the receptor protein-tyrosine phosphatases RPTPalpha, LAR, RPTPmu and CD45. FEBS J. 2008;275(10):2597-2604.

63. Cross JV, Templeton DJ. Regulation of signal transduction through protein cysteine oxidation. Antioxid Redox Signal. 2006;8(9-10):1819-1827.

64. Rhee SG, Bae YS, Lee SR, Kwon J. Hydrogen peroxide: a key messenger that modulates protein phosphorylation through cysteine oxidation. Sci STKE. 2000;2000(53):e1.

65. Hordijk PL. Regulation of NADPH oxidases: the role of Rac proteins. Circ Res. 2006;98(4):453-462.

66. van Wetering S, van Buul JD, Quik S, et al. Reactive oxygen species mediate Rac-induced loss of cell-cell adhesion in primary human endothelial cells. J Cell Sci. 2002;115(Pt 9):1837-1846.

67. Frank GD, Motley ED, Inagami T, Eguchi S. PYK2/CAKbeta represents a redox-sensitive tyrosine kinase in vascular smooth muscle cells. Biochem Biophys Res Commun. 2000;270(3):761-765.

68. Bokoch GM, Knaus UG. NADPH oxidases: not just for leukocytes anymore! Trends Biochem Sci. 2003;28(9):502-508. 
69. Seshiah PN, Weber DS, Rocic P, Valppu L, Taniyama Y, Griendling KK. Angiotensin II stimulation of $\mathrm{NAD}(\mathrm{P}) \mathrm{H}$ oxidase activity: upstream mediators. Circ Res. 2002;91(5):406-413.

70. van Buul JD, Fernandez-Borja M, Anthony EC, Hordijk PL. Expression and localization of NOX2 and NOX4 in primary human endothelial cells. Antioxid Redox Signal. 2005;7(3-4):308-317.
71. Chen K, Kirber MT, Xiao H, Yang Y, Keaney JF Jr. Regulation of ROS signal transduction by NADPH oxidase 4 localization. $J$ Cell Biol. 2008;181(7):1129-1139.

72. Taddei A, Giampietro C, Conti A, et al. Endothelial adherens junctions control tight junctions by VE-cadherin-mediated upregulation of claudin-5. Nat Cell Biol. 2008;10(8):923-934.

\section{Publish your work in this journal}

Cell Health and Cytoskeleton is an international, peer-reviewed open access journal focusing on all aspects of cell structure and function contributing to normal physiology and cell health and exploring the pathogenesis of cell dysfunction leading to adverse conditions and disease in the organism. The journal welcomes papers covering original research,

\section{Dovepress}

basic science, reviews and evaluations, guidelines, expert opinion and commentary, case reports and extended reports. The manuscript management system is completely online and includes a very quick and fair peerreview system, which is all easy to use. Visit http://www.dovepress.com/ testimonials.php to read real quotes from published authors.

Submit your manuscript here: http://www.dovepress.com/cell-health-and-cytoskeleton-journal 